\title{
Plasma Expansion From a Radio Frequency Microdischarge
}

\author{
Rod Boswell, Christine Charles, Peter Alexander, James Dedrick, and Kazunori Takahashi
}

\begin{abstract}
Plasma expansion of a capacitively coupled radio frequency $(13.56 \mathrm{MHz})$ argon microdischarge into a larger glass tube is studied by acquisition and analysis of high-resolution digital images. The microdischarge operating conditions are a flow of about $1.5 \mathrm{~L} / \mathrm{min}$, a pressure of 7 torr, and a power of $10 \mathrm{~W}$. Interesting plume patterns can be generated, and an example of plasma striations within the plume is shown.
\end{abstract}

Index Terms-Aerospace engineering, plasma sources.

$\mathbf{O}$ VER the past few decades, there has been an increasing interest in the development of plasma discharges for electric propulsion in space [1], [2]. Propulsion systems vary with the type of missions (thrust and specific impulse requirements and power allocation). Ion acceleration can be obtained by plasma expansion. At low pressure (less than a few millitorr), large plasma volumes can be accelerated, e.g., ion acceleration to supersonic velocities in the helicon double layer thruster [3], and high-power operation can be envisaged. At high pressure, plasma volumes are smaller, but dense plasmas can be obtained with lower power input. Small-scale discharges can be used for microplasma propulsion [1].

Here, a capacitive radio frequency (RF) $(13.56 \mathrm{MHz}) \mathrm{mi}-$ crodischarge about $2 \mathrm{~cm}$ long and $1.5 \mathrm{~mm}$ in diameter known as "Pocket Rocket" [4] is expanded in a 12-cm-long 5-cmdiameter glass tube contiguously attached to a $30-\mathrm{cm}-$ long 16-cm-diameter aluminum vacuum chamber equipped with a primary pump and a baratron gauge. Argon gas is introduced upstream of the microdischarge into a small cavity or plenum $(1.2 \mathrm{~cm}$ wide and $4 \mathrm{~cm}$ in diameter) equipped with a convectron gauge. The plasma is coupled at 7 torr with about $10-\mathrm{W}$ RF power using a pi matching network.

It has been previously shown that $\mathrm{RF}$ breakdown requires less voltage than dc breakdown [5], and here, a peak-to-peak voltage of $500 \mathrm{~V}$ is used which gives a $\mathrm{pD}$ factor of about 1 torr $\cdot \mathrm{cm}$, which is not far from the Paschen minimum. These conditions

Manuscript received November 10, 2010; revised March 31, 2011; accepted April 10, 2011. Date of publication May 19, 2011; date of current version November 9, 2011.

R. Boswell, C. Charles, P. Alexander, and J. Dedrick are with the Space Plasma, Power and Propulsion Laboratory, Research School of Physics and Engineering, Australian National University, Canberra, A.C.T. 0200, Australia.

K. Takahashi is with the Space Plasma, Power and Propulsion Laboratory, Research School of Physics and Engineering, Australian National University, Canberra, A.C.T. 0200, Australia, and also with Iwate University, Department of Electrical and Electronic Engineering, Morioka 020-8551, Japan.

Digital Object Identifier 10.1109/TPS.2011.2143434 are monitored using a Casio EX-F1 digital camera, and a typical discharge from Pocket Rocket is shown in Fig. 1. Fig. 1(a) shows the plasma plume expansion from the microdischarge into the glass cavity, and Fig. 1(b) shows the plume in the vicinity of the extraction aperture.

For improved visualization of the plasma, a piece of black carbon paper, which is nonintrusive to the plasma and is typically used as an electrode in hydrogen fuel cells [6], has been fitted around the aperture to minimize reflections from the plenum. In Fig. 1(a), the plasma is observed to exit the aperture as a high-intensity stream and is propelled downstream towards the vacuum chamber in a collimated beam. The beam remains in this form for approximately two thirds of the tube, and its diameter decreases slightly with axial length for approximately $3 \mathrm{~cm}$ and then becomes divergent. The small region of increased luminosity underneath the stream exiting the aperture is a reflection from the plenum through a hole in the carbon paper.

In addition to the beam, a low-intensity diffuse plasma with an approximately hemispherical profile is evident at the beginning of the expansion section and reaches $3-4 \mathrm{~cm}$ into the tube. A similar effect is observed at the exit towards the vacuum chamber as the stream expands rapidly in the radial direction. In both of these cases, the plasma is in contact with the grounded plenum and diffusion chamber, respectively, and further investigation is underway to determine whether this boundary condition is influential to the behavior of the discharge.

For shorter exposure times of a few hundred milliseconds, the detailed structure of the plasma stream may be visualized as shown in Fig. 1(b). The luminosity of the diffuse lower density plume is lower than that of the stream exiting the aperture and is not visible here. A couple of millimeters from the aperture, a series of approximately spherical striations is present within the directed discharge stream, and these are also visible to the naked eye. The structures are spaced relatively equally at a pitch of about $5 \mathrm{~mm}$ and fill the full beam cross section in each case. The more luminous point occurring downstream of the striations is due to a reflection from the plenum rim. Although not well understood, striations have been reported before, e.g., in low-pressure RF plasmas [7] and in low-pressure dc plasmas [8].

Plasma generation may be either via gamma electrons or capacitively coupled sheath heating mechanism. The contribution of the plasma to the thrust is being investigated. It is likely that the plasma heats the propellant, and the resulting thrust would be larger than the thrust generated by the gas stream alone. This device could then be used as an RF microthruster for space applications [4]. 


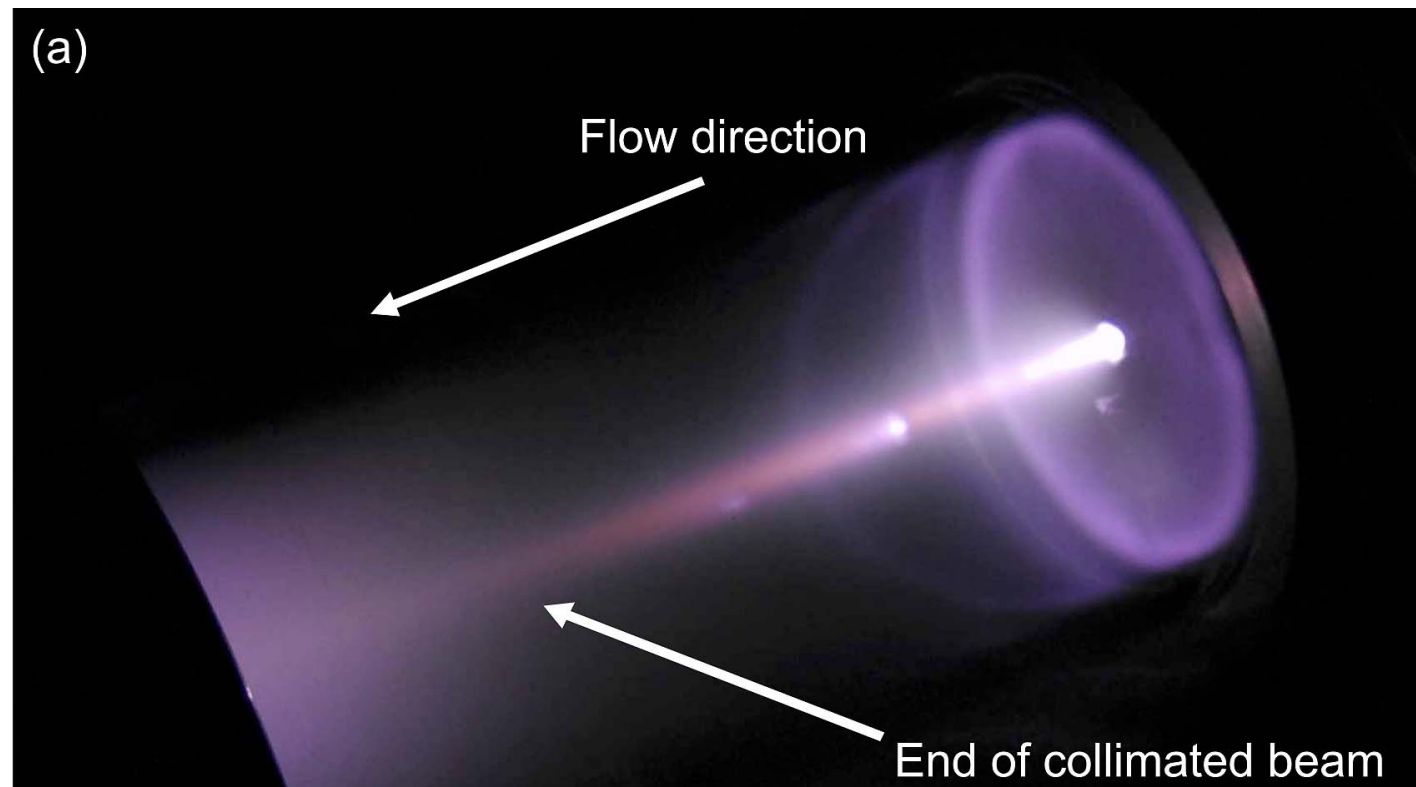

Fig. 1. Photograph of (a) Pocket Rocket showing the plasma stream exiting the microdischarge and (b) a shorter exposure time example of the microdischarge showing striations.

\section{REFERENCES}

[1] D. M. Goebel and I. Katz, Fundamentals of Electric Propulsion. Hoboken, NJ: Wiley, 2008.

[2] C. Charles, "Plasmas for spacecraft propulsion," J. Phys. D, Appl. Phys., vol. 42, no. 16, p. 163001 , Aug. 2009.

[3] C. Charles, "A review of recent laboratory double layer experiments," Plasma Sources Sci. Technol., vol. 16, no. 4, pp. R1-R25, Nov. 2007.

[4] lodged Oct. 26, 2010 R. W. Boswell, "RF micro-thruster for space applications," ANU Provisional Patent Application.

[5] H. B. Smith, C. Charles, and R. W. Boswell, "Breakdown behavior in radiofrequency argon discharges," Phys. Plasmas, vol. 10, no. 3, pp. 875-881, Mar. 2003.
[6] A. Caillard, P. Brault, J. Mathias, C. Charles, and R. W. Boswell, "Deposition and diffusion of platinum nanoparticles in porous carbon assisted by plasma sputtering," Surf. Coat. Technol., vol. 200, no. 1-4, pp. 391-394, Oct. 2005.

[7] H. C. J. Mulders, W. J. M. Brok, and W. W. Stoffels, "Striations in a lowpressure RF-driven argon plasma," IEEE Trans. Plasma Sci., vol. 36, no. 4, pp. 1380-1381, Aug. 2008.

[8] H. Ghomi, S. Sadrieyeh, and N. N. Safa, "A simple explanation of Schottky conditions through striations," IEEE Trans. Plasma Sci., vol. 36, no. 4, pp. 1004-1005, Aug. 2008. 